\title{
Ohana: detecting selection in multiple populations by modelling ancestral admixture components
}

\author{
Jade Yu Cheng, ${ }^{*}, 1,2$ Fernando Racimo, ${ }^{1}$ Rasmus Nielsen, ${ }^{1,2}$ \\ ${ }^{1}$ Centre for GeoGenetics, Natural History Museum of Denmark, University of Copenhagen, Oster Voldgade 5-7, \\ Copenhagen 1350 Denmark \\ ${ }^{2}$ Departments of Integrative Biology and Statistics, University of California, Berkeley, Berkeley, CA 94720, USA \\ *Corresponding author: E-mail: xxx@xxx. \\ Associate Editor: xxx \\ The xxx data were obtained from $\mathrm{xxx}$ (accession no. $\mathrm{xxxx}$ ).
}

\begin{abstract}
One of the most powerful and commonly used methods for detecting local adaptation in the genome is the identification of extreme allele frequency differences between populations. In this paper, we present a new maximum likelihood method for finding regions under positive selection. The method is based on a Gaussian approximation to allele frequency changes and it incorporates admixture between populations. The method can analyze multiple populations simultaneously and retains power to detect selection signatures specific to ancestry components that are not representative of any extant populations. We evaluate the method using simulated data and compare it to related methods based on summary statistics. We also apply it to human genomic data and identify loci with extreme genetic differentiation between major geographic groups. Many of the genes identified are previously known selected loci relating to hair pigmentation and morphology, skin and eye pigmentation. We also identify new candidate regions, including various selected loci in the Native American component of admixed Mexican-Americans. These involve diverse biological functions, like immunity, fat distribution, food intake, vision and hair development.
\end{abstract}

Key words: Positive selection, admixture, population structure, human evolution, selective sweeps

\section{Introduction}

The emergence of population genomic data has facilitated fine-scale detection of regions under recent positive selection in humans and other species. There are multiple different methods for carrying out such selection scans. Some of these methods rely on patterns of long-range linkagedisequilibrium (Sabeti et al., 2007;Voight et al.,
2006), one of the characteristic genomic footprints left by a selective sweep (Kim and Stephan , 2002;Kim and Nielsen , 2004;McVean , 2007). However, this pattern fades rapidly over time, and these methods are, consequently, best suited for detecting very recent selective sweeps from de novo mutations. Other methods, based on distortions in the allele frequency spectrum caused by positive selection, can allow for the detection of more ancient events, but are generally only 


$$
\text { "output" — 2019/2/14 - 3:53 — page } 2 \text { - \#2 }
$$

Cheng et al. · doi:10.1093/molbev/mst012

MBE

applicable one population at a time (Tajima , 1989;Fu and Li , 1993;Fay and Wu , 2000;Nielsen , 2005;Huber et al., 2016;DeGiorgio et al., 2016).

A different class of methods for detecting selection analyses patterns of allele frequency differentiation between populations. These methods proceed, for example, by computing Wright's fixation index $\left(F_{S T}\right)$ locally across different regions of a genome(Beaumont and Nichols , 1996;Akey et al., 2002;Beaumont and Balding , 2004). The basic idea is that regions that have experienced episodes of positive selection will display frequency differences between populations that are stronger than what would be expected under pure genetic drift. Population differentiation methods can detect more ancient selective events than linkage disequilibrium-based methods (Sabeti et al., 2006), and are sensitive to different types of positive selection events, including sweeps from a de novo mutation, sweeps from standing variation, incomplete sweeps, and adaptive introgression (Yi et al., 2010;Bonhomme et al., 2010;Fumagalli et al., 2015;Racimo et al., 2016). Recent methods have allowed researchers to detect excess local differentiation on particular branches of a 3-population tree (Yi et al., 2010;Racimo , 2016), a 4-population tree (Cheng et al., 2017) or an abitrarily large tree (Librado and Orlando , 2018), albeit without modeling post-split admixture events.

A generalization of these methods was developed by (Gnther and Coop , 2013;Gautier
, 2015;Coop et al., 2010). Their method can handle an arbitrary number of populations and detects positive selection as a genomically local distortions from a genome-wide covariance matrix, which is used as a neutral baseline. Similar methods have used hierarchical Bayesian models (Foll and Gaggiotti, 2008;Foll et al., 2014) or principal component analysis (Duforet-Frebourg et al., 2015) to model patterns of population differentiation to identify local distortions across the genome. Another method ((Fariello et al., 2013)) extended single-locus differentiationbased methods to the analysis of haplotype differentiation. More recently, Mathieson et al. (2015) developed an admixture-aware selection test based on a linear model and applied it to human data. The analysis took advantage of the fact that present-day European populations could be modeled as a mixture of three highly differentiated ancestral components. Regions of the genome that exhibited strong deviations from the genome-wide mixture proportions were therefore strong candidates for positive selection. Finally, Refoyo-Martinez et al. (2018) developed a method to test for selection on an admixture graph, which represents the history of divergence and admixture events among populations. Although useful for detecting selection in the presence of admixture, it still requires the user to specify which individuals belong to which populations, and to infer the graph in advance. 


$$
\text { "output" — 2019/2/14 - 3:53 — page } 3 \text { - \#3 }
$$

Ohana $\cdot$ doi:10.1093/molbev/mst012

MBE

Here, we introduce a new selection detection framework that can explicitly model admixture and detect selection from populations of admixed ancestries. It can simultaneously compare arbitrarily many populations and ancestry components and is encoded in a flexible framework for testing selection on a specific lineage or set of lineages. The method allows the user to identify signals of positive selection via population differentiation, without relying on self-reported ancestry or admixture correction to group individuals into populations. The method can also determine if a selective event is specific to a particular population or shared among different populations.

Unlike previous methods, we fully take advantage of admixed populations, and we do not require the user to a priori categorize samples into populations, or to correct allele frequencies to account for recent admixture. Thus, the selection scan does not rely on user-supplied sample labels or ancestry compositions. The methods identifies positive selection by searching for loci showing distortions in the population covariance matrix, relative to the genome-wide baseline. It provides a flexible framework to specifically test for selection on individual components or sets of components. This functionality allows researchers to accommodate specific evolutionary scenarios into the range of testable hypotheses, including local adaptation, adaptive introgression, and convergent selection. The method first coestimates the population structure of the input panel and the allele frequencies of the ancestral admixture compomnents through an unsupervised learning process (Cheng et al., 2017), before testing for selection on the ancestral components themselves. Researchers can also use the method to examine estimated population structure and visualize trees connecting the ancestral components using plotting functionalities provided by our software package, Ohana, as part of the analysis pipeline.

\section{Methods}

\section{Basic model}

The new method is based on the Ohana inference framework (Cheng et al., 2017), which works with both genotype calls and genotype likelihoods. In brief, the classical Structure model (Pritchard et al., 2000) is used to infer allele frequencies, ancestry components, and admixture proportions using maximum likelihood (ML). Then a covariance matrix among components is inferred using a multivariate Gaussian distribution while enforcing constraints imposed by the assumption of a tree structure. This system is underdetermined (see e.g., Felsenstein (1985)), i.e. multiple covariance matrices induce the same probability distribution on the allele frequencies. To circumvent this issue, we root the tree in one of the ancestry components. This corresponds to conditioning on the allele frequencies in one of the components 


$$
\text { "output" — 2019/2/14 - 3:53 — page } 4 \text { - \#4 }
$$

Cheng et al. · doi:10.1093/molbev/mst012

MBE

when calculating the joint distribution of allele frequencies in the other components. This idea is similar to Felsenstein's restricted maximum likelihood approach (Felsenstein, 1985). We emphasize that the rooting is arbitrary but that it does not imply any assumptions about this component actually being ancestral.

Through ML estimation we obtain the covariance matrix $\Omega^{\prime}$, which has size $(K-1) \times(K-1)$ and a joint density shown in Eq. 1, where $f_{k j}$ is the estimated allele frequency for ancestry component $k$ at $\operatorname{SNP} j$ and $\mu_{j}$ is the sample allele frequency for $\operatorname{SNP} j$, obtained either by counting alleles in the case of called genotypes or by EM estimation in the case of genotype likelihoods (Cheng et al., 2017).

$$
\begin{aligned}
\ln \left[P_{2}(F)\right] & =\ln \left\{\prod_{j}^{J}\left[\frac{1}{\sqrt{\left|2 \pi c_{j} \Omega^{\prime}\right|}} \exp \left(-\frac{1}{2} \cdot f_{j}^{\prime T} \cdot\left(c_{j} \Omega^{\prime}\right)^{-1} \cdot f_{j}^{\prime}\right)\right]\right\} \\
& =-\frac{1}{2} \cdot \sum_{j}^{J}\left\{(K-1) \cdot \ln \left(2 \pi c_{j}\right)+\ln \left[\operatorname{det}\left(\Omega^{\prime}\right)\right]+\frac{1}{c_{j}} \cdot f_{j}^{\prime T} \cdot \Omega^{\prime-1} \cdot f_{j}^{\prime}\right\} \\
\text { where } c_{j} & =\mu_{j}\left(1-\mu_{j}\right) \\
f_{j}^{\prime} & =f_{j}-f_{j_{0}}
\end{aligned}
$$

Following the structure analysis and component tree inference, a natural extension of this framework is to detect SNPs that deviate strongly from the globally estimated covariance structure. The idea of testing for deviations from a Gaussian distribution follows (Gnther and Coop , 2013), but differs in the use of an enforced treestructure, an ML inference framework and fast optimization algorithms, thereby avoiding some of the computational challenges associated with
Markov Chain Monte Carlo (MCMC). We also incorporate admixture into our model, thereby enabling the possibility to test for positive selection acting on the ancestral components of a panel, before more recent admixture occurred between the ancestors of the sampled individuals Selection model

The test for selection is based on a likelihood ratio test that identifies SNPs with allele frequency patterns that are poorly described by the genome-wide covariance pattern. A genome-wide covariance matrix is estimated from all SNPS jointly. Each SNP is then independently tested for deviations from this model, using a scalar factor introduced to certain elements of the covariance matrix. This scalar factor can be introduced in different ways depending on which selection hypotheses are tested. In our analyses, we chose to scale the covariance matrix such that one of its diagonal values is multiplied by a scalar, $\alpha$, corresponding to strong differences in allele frequency in one of the ancestry components relative to the rest:

$$
P\left(f_{j} \mid \Omega, \mu_{j}\right) \sim \mathcal{N}\left(\mu_{j}, \mu_{j}\left(1-\mu_{j}\right)\left[\begin{array}{ccc}
\Omega_{0,0} & \cdots & \Omega_{0, k-1} \\
\vdots & & \vdots \\
\Omega_{k-1,0} & \cdots & \alpha \cdot \Omega_{0, k-1}
\end{array}\right]\right)
$$

The value of $\alpha$ is then estimated using ML and a likelihood ratio is formed by testing the hypothesis of $\alpha=1$ against the alternative of $\alpha>$ 1. A high likelihood ratio indicates a larger deviation in allele frequency in a focal component 


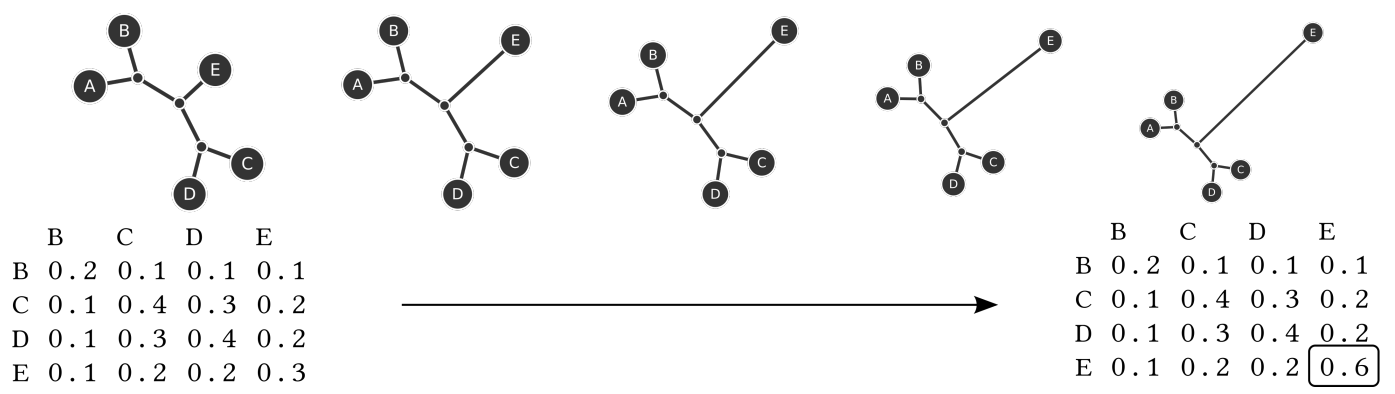

FIG. 1. Selection hypotheses and their encodings as covariance matrices. In this example, the ancestry component E is assumed to be the potential target of selection. The entry $\mathrm{E}: \mathrm{E}$ in the covariance matrix is therefore allowed to deviate from the globally estimated value.

than expected under the globally estimated nullmodel. Figure 1 shows an example. This test can also be implemented to test selection on ancestral non-terminal lineages by multiplying the corresponding values in the covariance matrix by a scaling factor.

Under the null-hypothesis, the likelihood ratio test statistic is expected to follow a 50:50 mixture between a $\chi_{1}^{2}$-distribution and a point mass at zero (Self and Liang, 1987).

In summary, we estimate a scaling factor for one or more components of the covariance matrix in a multivariate normal model of allele frequency distribution among populations. For each candidate SNP, we then compare the estimated covariance matrix to that obtained genome-wide, using a likelihood ratio test.

\section{Optimization}

For ML-based population structure inference, we use an optimization algorithm based on an Active Set method (Murty and Yu , 1988) to solve the sequential quadratic programming problem. This method was previously shown to have better computational performance than competing methods (Cheng et al., 2017). For the ML-based ancestry covariance estimates, we use the Nelder-Mead simplex method (Nelder and Mead , 1965). It uses Cholesky decomposition (Cholesky , 1910) to determine the positive semidefiniteness of a matrix and to compute matrix inverses and determinants. For identifying the best local covariance structure during a selection scan, we use a simple Golden-section search algorithm (Kiefer , 1953) to find the solution for the single scalar multiplier associated with a specific selection hypothesis.

Simulations

To evaluate the performance of the methods, we generate simulations using msms (Ewing and Hermisson , 2010) under specific demographic models and specific tree structures (Figure 2). We focus on multi-population demographics that are simulated in a tree-like fashion with positive selection events occurring in either all or some of the branches leading to present-day samples.

Specifically, we simulate an effective population size $N_{e}$ of 10,000 for all populations, and obtain 20 chromosomes for each population. We use a 


$$
\text { "output" — 2019/2/14 - 3:53 — page } 6 \text { - \#6 }
$$

Cheng et al. · doi:10.1093/molbev/mst012

$\mathrm{MBE}$

population-scaled mutation rate $\theta=4 N_{e} \mu$ of 100 , a recombination rate $\rho=4 N_{e} r$ of 100 , with an finite cut site model as implemented in MSMS. We simulate 4 populations with population splits at $0.02,0.05$, and 0.12 coalescent units in the past (in units of $4 N_{e}$ ). This is illustrated in Figure 2.

We assessed the power of our selection test using simulations. We simulated 1,200 bp sequences where a single beneficial SNP located in the middle of the sequence is under direct positive selection. We also simulated 1,200 bp neutral sequences. We set the start of selection 0.02 coalescent units ago, and set the initial frequency of the beneficial allele at 0 (i.e. we simulate a de novo mutation). We assumed an additive model of fitness with scaled selection coefficient $2 N_{e} s$ ranging from 200 to 1000 for alleles in the homozygous state. In our model, the fitness of the heterozygote is $1+s / 2$ and the two homozygous fitnesses are 1 and and $1+s$. We set the forward mutation rate, $4 N_{e} \mu^{\prime}$, to 0.1 for the selected allele for mutations from the wild type to the selected type (the backwards mutation rate to the wild type is 0 ). In the simulations used for Figure 5, we combined 2 neutral sequences flanking a selected sequence. In the simulations used for Figure 4, we placed 10 neutral sequences flanking a selected sequence on either side.

A population can then be purely formed by one ancestry component, or as a mixture of several components. We generate un-admixed or admixed genotypic data by simulating the ancestry
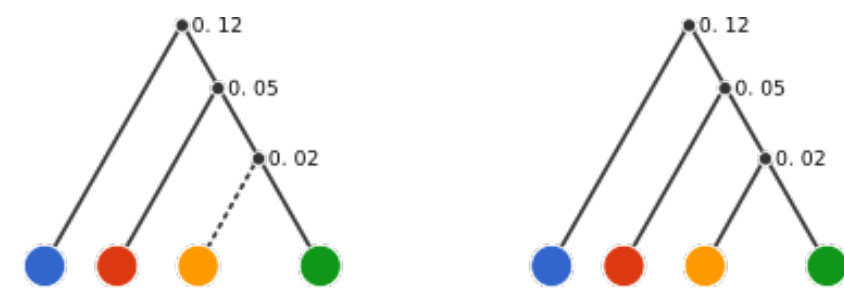

FIG. 2. Trees used for simulations. We simulated selection only on the yellow branch (left) and also simulated neutral segments according to the same tree but with no selection on any branches (right)

proportions, $Q$, for each individual (Figure 3). For un-admixed samples, we simply assign them to have $100 \%$ ancestry from one population. For admixed samples, we simulate equal admixture (in expectation) using the Dirichlet distribution, $\operatorname{Dir}(\alpha)$, where $\alpha=(1.0, \cdots, 1.0)$. Figure 3 illustrates these two admixture setups. In the mixed case, each population is (in expectation) an equal mixture of three of the ancestry components, i.e. each population lacks one of the four components.

We then sampled genotype observations under the assumption of independence, i.e. $p_{i j}^{A A}=$ $f_{i j}^{2}, p_{i j}^{A a}=2 f_{i j}\left(1-f_{i j}\right), p_{i j}^{a a}=\left(1-f_{i j}\right)^{2}$, where $f_{i j}=$ $\sum_{k} Q_{i k} F_{k j}$ is the allele frequency in locus $j$ for individual $i, Q_{i k}$ is the ancestry proportion of component $k$ in individual $i, F_{k j}$ is the allele frequency in ancestry component $k$ in locus $j$, and $p_{A A}, p_{A a}$ and $p_{a a}$ are the probabilities of observing major-major, major-minor, or minorminor genotypes for the locus, respectively. $F$ has dimensionality $K \times J$ and $Q$ has dimensionality $I \times K$, where $K$ is the number of ancestry components, $I$ is the number of samples, and $J$ is the number of SNPs. 


$$
\text { "output" — 2019/2/14 — 3:53 — page } 7 \text { — \# }
$$

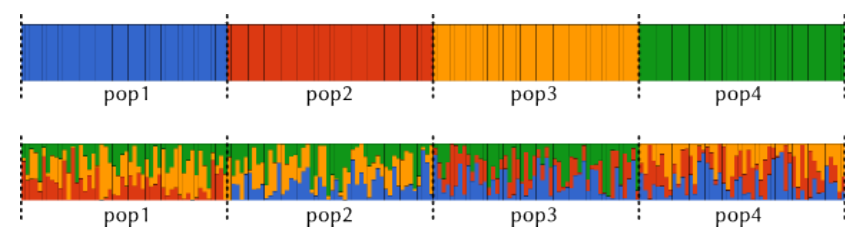

FIG. 3. Simulated admixture proportions for either unadmixed individuals (top) or equal admixture from three out of four ancestry components (bottom). In both scenarios, we simulated 4 populations of 20 individuals per population.

\section{Results}

\section{Simulations}

We first evaluated the efficacy of the method for fine-mapping the true selected allele.

We simulated 1000 replicates of 4 admixed populations and scanned the simulated genomes using our likelihood ratio test. As a measure of accuracy, we used the distance between the SNP with the highest likelihood ratio and the SNP under selection in the simulations. In the majority of simulations, the distance between the true and the inferred SNP is small, i.e. $<10 \%$ of sequence length, suggesting a generally high accuracy for fine-mapping.

We then measured the excess of false positive results under the null hypothesis of no selection (Figure 4). To do so, we first generated simulations under the null scenario (no selection, Figure 4top) and a scenario of positive selection affecting a particular ancestry branch (Figure 4-bottom). We also simulated 2 types of samples: un-admixed (left) and admixed (right). We computed perSNP likelihood ratios along the simulated genomes using the correct selection model and converted them to p-values. We then compared these pvalues in a quantile-quantile (QQ) plots against

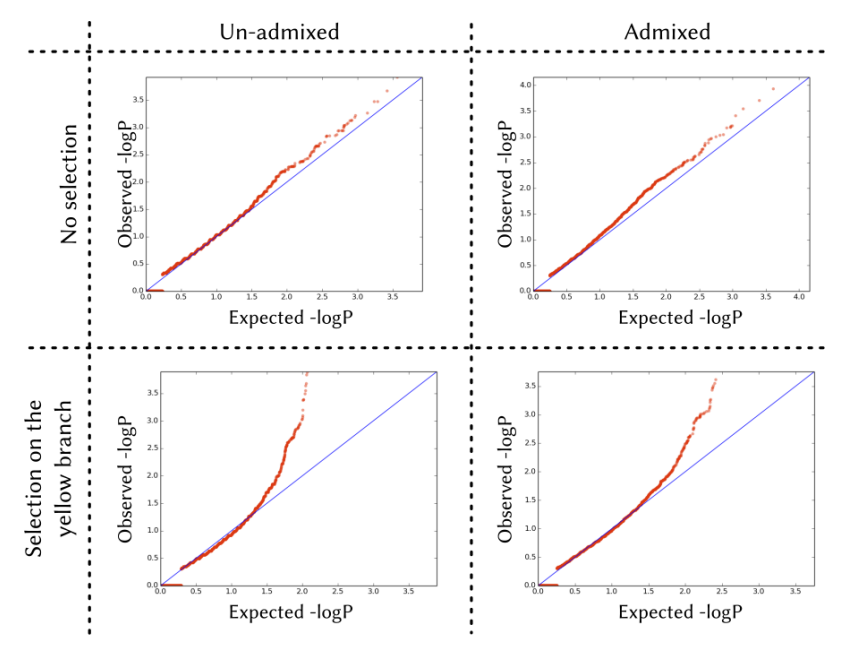

FIG. 4. Test for excess of false positive. We simulate 4 different scenarios: no selection (top) and selection on a single branch (bottom), assuming the input populations are un-admixed (left) and admixed (right). Statistically significant outliers are detected only when the data were simulated under the scenarios with selection.

a 50:50 mixture of values equal to 1 and random values sampled from a uniform distribution between 0 and 1 . This mixture corresponds to the expected distribution of $\mathrm{P}$-values of our statistic under the null model. In simulations with selection, deviations from the neutral expectation are visible (Figure 4-bottom), while no excess of false positives (elevated Type I errors) are present when populations are simulated without selection (Figure 4-top).

We then compared our method to three summary statistics: $F_{S T}$ (Wright et al., 1949; Weir et al., 2005), PBS (Yi et al., 2010), and FLK (Bonhomme et al., 2010) (Figure 5). As before, we generated simulations under 2 types of sample admixture, and simulated positive selection on the yellow component only. We tested for selection using 9 methods: Ohana, $2 F_{S T}$-based tests statistics, 2 PBS-based tests, and 4 FLK-based tests. The Ohana method was run so as to test 


$$
\text { "output" — 2019/2/14 - 3:53 - page } 8-\# 8
$$

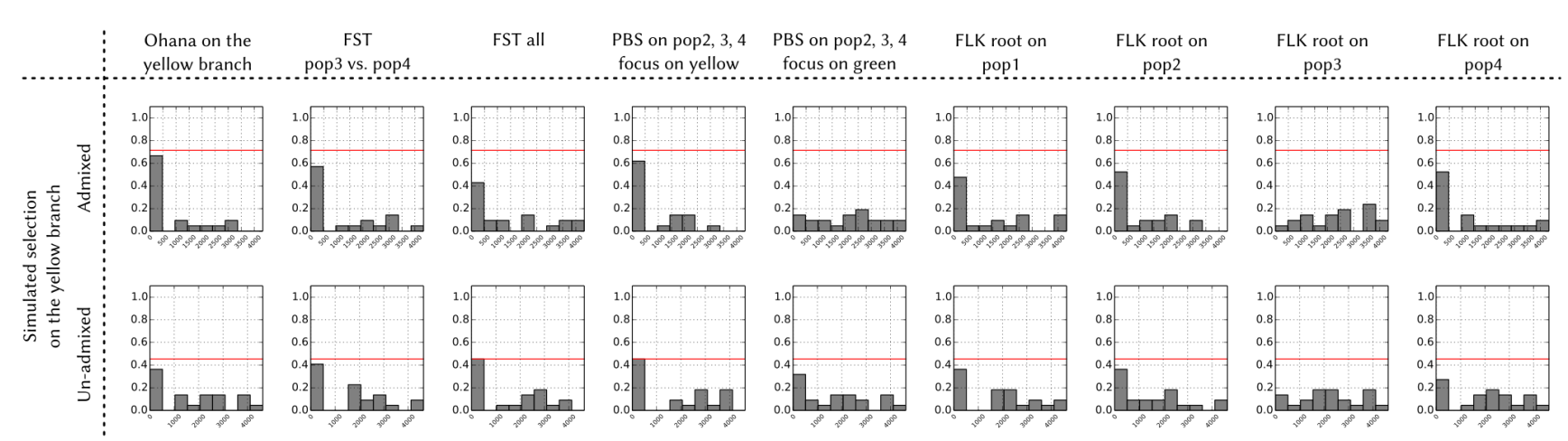

FIG. 5. Comparison of performance of selection tests. We simulated individuals that were either admixed (row 1) or unadmixed (row 2), as described in Figure 3. We then simulated selection on a single branch (yellow). We simulated the $\mathrm{SNP}$ in the midpoint location to be under direct selection, with a selection strength of $2 N_{e} s=600$ for the homozygote and $2 N_{e} s=300$ for the heterozygote. We then scanned for selection signals using Ohana (first column). We calculated the FST statistics in 2 ways: FST between two population (one with the yellow component one without), and FST among all four populations. We also calculated the PBS statistics in 2 ways: yellow-specific or green-specific. Finally, we calculated the FLK statistics in 4 ways by specifying each of the 4 populations as the outgroup. In admixed simulations, Ohana outperforms the rest of the tests by achieving a higher proportion of simulations in which the simulated SNP under selection is $<120 b p$ (10\% of total sequence length) from the SNP with the highest test score.

specifically for selection on the yellow-branch. In one of the $F_{S T}$-based tests, we calculated pairwise $F_{S T}$ between 2 populations: one containing the yellow component and one not containing it. In the second $F_{S T}$-based test, we calculated $F_{S T}$ among all 4 populations. In the 2 PBS-based tests, we tested for selection focusing on either the yellow or the green component as the "target" branch. In the 4 FLK-based tests, we specified each of the 4 populations as the outgroup in turn.

The performance of the methods was measured by the percentage of runs in which the simulated and detected SNP are within $10 \%$ of the total sequence length. In admixed samples, our method achieves the best outcome when the proper model is specified (Figure 5 row 1). When analyzing purely un-admixed samples, our method is on-par with the best method among all $F_{S T}$, PBS, and FLK tests (Figure 5 row 2).

We then compared all methods in Figure 5 using 2 measures: the percentage of times that the selection method identifies the simulated causal SNP as the top SNP (Table 1) and the mean distance in bp between the simulated causal SNP and the top SNP identified by the selection method (Table 1 and 2).

For all methods, scenarios with admixed samples lead to weaker performance than in their un-admixed counterparts. In un-admixed cases, our method performs equally or better than summary statistics. In admixed cases, our method reaches higher accuracy than other methods. For example, in the admixed yellow-branch simulation when $2 N s=600$, Ohana identifies the selected allele $21.57 \%$ of the time, while this value is $14.95 \%$ for pairwise $F_{S T}, 8.09 \%$ for global $F_{S T}$, $18.63 \%$ for PBS, and $7.11 \%$ for FLK.

Analysis of real data

We identified regions in the genome that are likely to have been under the influence of positive selection using a merged dataset containing several population panels from phase 3 of the 


$$
\text { "output" — 2019/2/14 - 3:53 — page } 9-\# 9
$$

Ohana $\cdot$ doi:10.1093/molbev/mst012

MBE

1000 Genomes Project (1000 Genomes Project Consortium , 2015). We randomly selected 64 genomes from each of 4 populations from the 1000 Genomes project: the British from Great Britain (GBR), the Han Chinese from Beijing (CHB), the Yoruba Africans (YRI) and the admixed Mexican-Americans from Los Angeles (MXL) (the number 64 was chosen because it was the size of the smallest panel). We only included variable sites with no missing data and a minimum allele frequency of 0.05 across the entire merged panel. In total, we analyzed 5,601,710 variable sites across the autosomal genome. We inferred genome-wide allele frequencies and covariances for the latent ancestry components as described in the Methods section, using $K=4$. To scan for covariance outliers, we performed four hypothesisdriven scans, in which we specifically searched for selection separately in each of the four inferred ancestry components in our dataset (Table 3).

After running these scans, we queried the CADD server (Rentzsch et al., 2018) to obtain functional, conservation and regulatory annotations for the top candidate SNPs, including SIFT (Sim et al., 2012), PolyPhen (Adzhubei et al., 2013), GERP (Davydov et al., 2010), PhastCons (Siepel et al., 2005), PhyloP (Pollard et al., 2010) and Segway (Hoffman et al., 2012) annotations, so as to find the changes most likely to be disruptive. We discuss some of these below. We also queried the GTEx cis-eQTL database (Lonsdale et al., 2013), the UK Biobank
GeneATLAS (Canela-Xandri et al., 2018), and the GWAS catalog (MacArthur et al., 2017), to look for trait-associated SNPs. We particularly focus on SNPs that have both high log-likelihood ratios in favor of positive selection $(L L R S>15)$ and high CADD scores in favor of functional disruption $(>10)$.

Below, we describe some of the top SNPs with high LLRS and their surrounding regions, for those cases in which available genic, expression or regulatory information can provide us some clue as to the possible organismal function that may have been affected by the selective event. We particularly focus on the Native American ancestry scan (Table S4, Figure 6), as few selection scans have been performed in this population, but also briefly summarize the results from the other scans.

European ancestry scan

Results for the top 30 loci in the European ancestry scan are presented in Table S1. Most loci have been previously shown to be under selection in Europeans populations, including SLC45A2, SLC24A5, BNC2, the OCA2/HERC2 region, the LCT/MCM6 region and the TLR region (Mathieson et al., 2015;Bersaglieri et al., 2004; Voight et al., 2006; Vernot et al., 2014;Barreiro et al., 2009). We notice that, in several cases, the presumed causal SNP previously identified in the literature coincides with the SNP with the strongest selection signal. This is the case, for example, for rs1426654 (SLC24A5) 


$$
\text { "output" — 2019/2/14 — 3:53 — page } 10-\# 10
$$

Cheng et al. · doi:10.1093/molbev/mst012

$\mathrm{MBE}$

(Kimura et al., 2009;Lamason et al., 2005) and for rs16891982 (SLC45A2) (Branicki et al., 2008).

This suggest that the top SNPs for other loci, for which the causal SNPs are not yet known, may be good candidates for further tests of functional effects.

East Asian ancestry scan

We also performed a scan where we sought to recover SNPs that were candidates for selection in the ancestry component that is prevalent among our East Asian samples. Results for the top 30 loci in this scan are in Table S2. Here, we also recover several candidate regions that have been previously reported in East Asian selection scans, including ABCC11, POU2F3, ADH1B, FADS1 and TARBP1 (Vernot et al., 2014;Liu et al., 2018; Ohashi et al., 2011;Peng et al., 2010;RefoyoMartinez et al., 2018). Here, as in the previous scan, the top-scoring SNPs also tend to have the strongest phenotypic associations. For example, the highest scoring SNP (rs17822931) is the well-known missense variant in ABCC11, which is involved in sweat and earwax production (Yoshiura et al., 2006).

Yoruba / ancestral non-African ancestry scan Because our algorithm relies on an unrooted ancestry tree, we cannot distinguish between SNPs under positive selection in the terminal branch leading to the Yoruba / Sub-Saharan Africans and the ancestral non-African branch (Table S3). Nevertheless, more careful study of the allele frequencies of these SNPs in other 10 populations may serve to distinguish among these scenarios in the future. As in the the other ancestry scans, we also retrieve several genes that have been previously reported in positive selection studies. For example, the highest-scoring SNP is a missense variant in SLC39A4 (rs1871534) that has been reported to be under selection in SubSaharan Africa and to be causal for zinc deficiency (Engelken et al., 2014).

Native American ancestry scan The Native American ancestry scan yielded several novel candidates for positive selection (Table S4). As this ancestry has been less studied than the other aforementioned populations in the selection scan literature, we decided to extensively describe the top 30 candidates in the Supplementary Notes. We also highlight some of the more interesting regions here.

The top SNP (rs140736443) is located in an intron of LINC00871. This SNP does not have a high CADD score $(=1.125)$, but is very close to a SNP (rs10133371) with a very high LLRS (= 16.54) and CADD score $(=15.99)$. This SNP is also intronic but is highly conserved in primates (PhastCons $=0.972)$ and is located in a GERP conserved element $(\mathrm{P}=1.92 \mathrm{e}-21)$. LINC00871 is a long non-coding RNA gene that has been associated with number of children born (Barban et al., 2016), although the specific trait-associated SNP in that study does not have a high LLRS. This gene also contains a suggestive association to longevity in females (Zeng et al., 2018), although 


$$
\text { "output" — 2019/2/14 — 3:53 — page } 11 \text { — \#11 }
$$

Ohana · doi:10.1093/molbev/mst012

MBE

this study was under-powered to retrieve genomewide significant associations.

The third top SNP (rs2316155) has a low CADD score $(=0.633)$ but is located near two SNPs with high LLRS (rs1466182, rs1466183) that overlap a regulatory region (ENSR00000088366) and have high CADD scores (= 16.8 and 19.5, respectively). Both of these SNPs have high PhastCons conservation scores across primates, mammals and vertebrates, and both overlap a GERP conserved element.

The sixth top SNP (rs10508343) has a low CADD score but lies very close to another SNP (rs17143255) with a high LLRS and a very high CADD score $(=14.16)$. The latter is an intergenic SNP overlapping a GERP conserved element between LINC00708 and GATA3, which has been shown to lead to abnormal hair shape and growth in mice when mutated (Kaufman et al., 2003). Interestingly, SNPs overlapping LINC00708 have been recently associated with hair shape in a GWAS of admixed Latin Americans (Adhikari et al. 2016). There is also a high-LLRS SNP in this region that is significantly associated with the response to treatment for acute lymphoblastic leukemia (rs10508343) (Yang et al., 2009).

The seventh top SNP (rs16959274) is a GTEx eQTL for GOLGA8A for tibial artery and skeletal muscle, and for GOLGA8B in pancreas. These two genes are members of the same gene family, and code for an auto-antigen localized in the surface of the Golgi complex (Eystathioy et al., 2000).
The tenth top SNP (rs12580697) is a GTEx eQTL for TMTC1 in whole blood and has a moderately high CADD score $(=8.676)$. TMTC1 codes for an endoplasmic reticulum transmembrane protein that is involved in calcium homeostasis (Sunryd et al., 2014).

The eleventh top SNP (rs75607199) has a low CADD score but lies near three other SNPs (rs41325445, rs4901738 and rs59250732) with almost equally high LLRS and high CADD scores (= 13.49, 19.7 and 12.67, respectively). All of these SNPs are intronic and overlap OTX2-AS1, a long non-coding RNA gene. The SNP with the highest CADD score (rs4901738) is located in a GERP conserved element and has high PhastCons conservation scores across primates and mammals (>0.98). They all lie upstream of OTX2, coding for a developmental transcription factor implicated in microphtalmia (Ragge et al., 2005), retinal dystrophy (Vincent et al., 2014) and pituitary hormone deficiency (Diaczok et al., 2008). In mice, this gene has been found to be involved in the embryonic development of the brain (Boncinelli et al., 1993), photoreceptor development (Nishida et al., 2003) and susceptibility to stress (Peña et al., 2017).

The fourteenth top SNP (rs78441257) has a fairly high CADD score $(=12.72)$ and lies in a GERP conserved element of the 3' UTR of LRAT. This gene is implicated in retinal dystrophy (Thompson et al., 2001) and retinitis pigmentosa (Sénéchal et al., 2006). 
The fifteenth top SNP (rs1919550) is a GTEx eQTL for FBXO40 in whole blood, but does not have a high CADD score. However, it lies near a SNP (rs9813391) with a high LLRS that leads to a nonsynonymous change (R145Q) in ARGFX a homeobox gene - and another SNP (rs4676737) with both a high LLRS and high CADD score (= 14.07) overlapping a repressor region in an intron of FBXO40. The latter SNP is a GTEx eQTL for IQCB1 in fibroblasts, muscular esophagus and thyroid. IQCB1 is associated with Senor-Loken syndrome (Otto et al., 2005), a ciliopathic eye disorder.

The twenty-second top SNP (rs4946567) is an eQTL of TBC1D32 in cerebellar brain. This SNP has a high CADD score $(=11.02)$ and is conserved across vertebrates (vertebrate PhyloP $=0.916$, vertebrate PhastCons $=0.747$ ) Interestingly, the region in which it is located also harbors signature of selection in Yucatan miniature pigs (Kim et al., 2015; Kwon et al., 2018). TBC1D32 plays a role in cilia assembly (Ko et al., 2010) and may be involved in ciliopathic congenital abnormalities, including midline cleft, microcephaly, and microphthalmia (Adly et al., 2014).

The twenty-third and twenty-fourth top SNPs (rs5758430, rs4822061) are close to each other and lie in a large region with several high-LLRS SNPs. They are both linked GTEx eQTLs to several genes in a variety of different tissues. They are also both significantly associated with several traits related to body fat, food intake and white blood cells in the UK Biobank GeneATLAS $\left(\mathrm{P}<10^{-8}\right.$, see Supplementary Notes). Although these SNPs do not have particularly high CADD scores, there are several neighboring linked high-LLRS, highCADD SNPs with significant associations to the same traits, including splice site and missense mutations (Supplementary Notes). We also find two significantly-associated SNPs in the GWAS catalog in this region $\left(\mathrm{P}<10^{-8}\right)$ : rs4822024 is associated with Vitiligo (Jin et al., 2012) and rs13054099 is associated with neuroticism (Nagel et al., 2018).

\section{Discussion}

We describe a new modeling framework that can detect signals of positive selection on ancestry components, using allele frequency patterns across admixed populations. It models admixture explicitly and works with an arbitrary number of populations with or without admixed ancestries. It also does not rely on labeling of samples into particular populations, and allows for testing of different positive selection models reflecting different historical adaptive hypotheses.

The run-time complexity of our method is linear in the number of markers, but we still recommend a high-performance cluster to be used in a typical genomic analysis. With parallelization, a selection scan takes $<10$ minutes to analyze a 6 Mbp genome for $<10$ ancestry components using 100 cores. An example of how to perform this 


$$
\text { "output" — 2019/2/14 — 3:53 — page } 13 \text { — \#13 }
$$

Ohana · doi:10.1093/molbev/mst012

MBE

parallelization can be found on the project's wiki page on GitHub.

Our method works by testing for selection in specific components of the ancestry covariance matrix. We also explored what would occur if we used a likelihood model in which the ancestry covariance matrix was multiplied by a scalar, so as to find "global" candidates for selection rather than testing for selection in particular ancestries. We found however, that this was not an optimal way to detect candidates for selection, as it is biased towards finding many variants in highly drifted populations, likely because the excess variance in the Wright-Fisher process is not well modelled by the multivariate Gaussian assumption, especially at the boundaries of fixation and extinction.

When specifically testing for candidates for selection in Europeans, East Asians and SubSaharan Africans we identified several well-known candidates under positive selection, including OCA2, SLC24A5, SLC45A2, ABCC1 and SLC39A4. Many of our top scoring SNPs were also previously known to be causal for particular traits, as in the case of rs17822931 in ABCC11 in East Asians, rs16891982 in SLC45A2 in Europeans, rs1426654 in SLC24A5 in Europeans and rs1871534 in SLC39A4 in Sub-Saharan Africans.

Our scan for positive selection in the Native American ancestry component of Latin Americans yielded several novel candidates for adaptation in the human past. We found signatures of selection near genes involved in fertility (LINC00871), hair shape and growth (LINC00708), immunity (GOLGA8A / GOLGA8B and IRAK4), vision (OTX2 and LRAT), the nervous system (MDGA2) and various ciliopathies (IQCB1 and TBC1D32). Several of the highest-scoring SNPs in the candidate regions are known to be cis-eQTLs to their nearby genes, as is the case for rs12580697 / TMTC1 (involved in calcium homestasis) and rs4676737 / IQCB1 (involved in ciliopathies). We also found individual SNPs with high likelihood ratio scores in favor of selection that are associated with a variety of phenotypes, including rs12426688 (fat percentage), rs10508343 (response to leukemia treatment), rs34670506 (insomnia), and the cluster of high-scoring SNPs that include rs5758430 and rs4822061, among other SNPs. This particular cluster is especially interesting, as the SNPs in the region are associated with a variety of traits related to body fat distribution, food intake and white blood cells, suggesting a possible underlying phenotype related to these traits that may have driven an adaptive event.

We provide a list of functional annotations for all the SNPs with high LLRS (>15) within a $2 \mathrm{Mb}$ region surrounding each of the top genome-wide SNPs, including CADD, conservation, regulatory and protein deleteriousness scores, which we hope will guide future functional validation studies in these regions of the genome (Table S5). 


$$
\text { "output" — 2019/2/14 — 3:53 — page } 14 \text { - \#14 }
$$

Cheng et al. · doi:10.1093/molbev/mst012

In conclusion, Ohana provides a fast and flexible selection-detection and hypothesis-testing framework. It is easy to use and has in-built visualization functionalities to explore patterns on a genome-wide and locus-specific scale. We believe it will be a useful tool for biologists aiming to study positive selection and understanding the genomic basis of adaptation, particularly in cases where demographic histories are complex or not well characterized. 


$$
\text { "output" — 2019/2/14 — 3:53 — page } 15 \text { — \#15 }
$$

Ohana · doi:10.1093/molbev/mst012

MBE

Table 1. We compared the new method with the $F_{S T}$, PBS, and FLK statistics. The data simulation and selection detection are as described in Figure 5. We quantify selection strength using the percentage of simulations among a total of 500 where the selection method accurately identifies the simulated causal SNP as the top SNP.

\begin{tabular}{|c|c|c|c|c|c|c|c|}
\hline $\begin{array}{l}\text { sample } \\
\text { admixing }\end{array}$ & $\begin{array}{l}2 \mathrm{Ns} \text { for the } \\
\text { homozygote }\end{array}$ & $\begin{array}{l}\text { Ohana } \\
\text { yellow }\end{array}$ & $\begin{array}{l}\text { FST } \\
2 \text { pop }\end{array}$ & $\begin{array}{l}\text { FST } \\
\text { all }\end{array}$ & $\begin{array}{l}\text { PBS } \\
\text { focus3 }\end{array}$ & $\begin{array}{l}\text { PBS } \\
\text { focus4 }\end{array}$ & $\begin{array}{l}\text { FLK } \\
\text { correct-outgroup }\end{array}$ \\
\hline & 200 & 0.00 & 0.00 & 0.00 & 0.00 & 0.00 & 0.00 \\
\hline & 300 & 0.00 & 0.00 & 0.00 & 0.00 & 0.00 & 0.00 \\
\hline & 400 & 0.00 & 4.76 & 0.00 & 0.00 & 0.00 & 0.00 \\
\hline & 500 & 4.76 & 0.00 & 4.76 & 9.52 & 0.00 & 9.52 \\
\hline \multirow[t]{9}{*}{ admixed } & 600 & 23.81 & 19.05 & 0.00 & 14.29 & 0.00 & 19.05 \\
\hline & 700 & 27.27 & 18.18 & 4.55 & 31.82 & 0.00 & 13.64 \\
\hline & 800 & 25.00 & 15.00 & 5.00 & 10.00 & 0.00 & 15.00 \\
\hline & 900 & 18.18 & 13.64 & 4.55 & 13.64 & 0.00 & 13.64 \\
\hline & 1000 & 24.00 & 16.00 & 8.00 & 20.00 & 0.00 & 12.00 \\
\hline & 200 & 0.00 & 0.00 & 0.00 & 0.00 & 0.00 & 0.00 \\
\hline & 300 & 0.00 & 0.00 & 0.00 & 0.00 & 0.00 & 0.00 \\
\hline & 400 & 4.55 & 4.55 & 4.55 & 4.55 & 0.00 & 0.00 \\
\hline & 500 & 22.73 & 18.18 & 13.64 & 18.18 & 0.00 & 0.00 \\
\hline \multirow[t]{5}{*}{ un-admixed } & 600 & 22.73 & 18.18 & 18.18 & 13.64 & 0.00 & 4.55 \\
\hline & 700 & 18.18 & 13.64 & 13.64 & 9.09 & 4.55 & 0.00 \\
\hline & 800 & 22.73 & 18.18 & 9.09 & 13.64 & 0.00 & 4.55 \\
\hline & 900 & 23.08 & 26.92 & 19.23 & 23.08 & 3.85 & 0.00 \\
\hline & 1000 & 31.82 & 31.82 & 31.82 & 31.82 & 0.00 & 22.73 \\
\hline
\end{tabular}

Table 2. Mean distance between the top-scoring SNP and the simulated beneficial SNP. We compare the new method with the $F_{S T}$, PBS and FLK statistics. The data simulation and selection detection methods are as described in Figure 5 .

\begin{tabular}{|c|c|c|c|c|c|c|c|}
\hline $\begin{array}{l}\text { sample } \\
\text { admixing }\end{array}$ & $\begin{array}{l}\text { 2Ns for the } \\
\text { homozygote }\end{array}$ & $\begin{array}{l}\text { Ohana } \\
\text { yellow }\end{array}$ & $\begin{array}{l}\text { FST } \\
2 \text { pop }\end{array}$ & $\begin{array}{l}\text { FST } \\
\text { all }\end{array}$ & $\begin{array}{l}\text { PBS } \\
\text { focus3 }\end{array}$ & $\begin{array}{l}\text { PBS } \\
\text { focus4 }\end{array}$ & $\begin{array}{l}\text { FLK } \\
\text { correct-outgroup }\end{array}$ \\
\hline & 200 & 2454.57 & 2066.76 & 1286.48 & 1768.67 & 1994.33 & 2024.95 \\
\hline & 300 & 1680.86 & 1978.95 & 1925.18 & 1760.95 & 2076.32 & 2540.00 \\
\hline & 400 & 1691.57 & 1923.48 & 2131.57 & 1925.10 & 2083.62 & 1934.52 \\
\hline & 500 & 1835.90 & 1626.48 & 1606.24 & 1567.19 & 2060.81 & 1591.05 \\
\hline \multirow[t]{9}{*}{ admixed } & 600 & 772.00 & 1189.62 & 1411.52 & 837.90 & 2117.86 & 1232.62 \\
\hline & 700 & 430.55 & 674.73 & 1483.50 & 700.18 & 1746.05 & 1006.91 \\
\hline & 800 & 873.25 & 1028.75 & 980.00 & 1123.05 & 1458.15 & 901.25 \\
\hline & 900 & 1067.09 & 903.73 & 1305.77 & 1145.05 & 1869.27 & 1435.09 \\
\hline & 1000 & 810.32 & 579.48 & 1118.32 & 572.92 & 2065.40 & 1158.68 \\
\hline & 200 & 2590.77 & 2272.14 & 2292.73 & 1642.50 & 2318.05 & 2315.73 \\
\hline & 300 & 2052.14 & 1763.05 & 2119.59 & 2142.18 & 1866.73 & 1781.64 \\
\hline & 400 & 1465.95 & 2031.00 & 2248.68 & 1369.36 & 2297.82 & 2401.82 \\
\hline & 500 & 1398.27 & 1386.36 & 1513.50 & 1211.09 & 1909.05 & 1768.45 \\
\hline \multirow[t]{5}{*}{ un-admixed } & 600 & 1669.77 & 1585.18 & 1541.36 & 1750.05 & 1795.41 & 1999.09 \\
\hline & 700 & 1191.82 & 1300.77 & 1325.23 & 1083.45 & 1934.05 & 1376.14 \\
\hline & 800 & 650.36 & 755.36 & 1022.95 & 652.82 & 1238.73 & 1087.50 \\
\hline & 900 & 558.62 & 602.88 & 710.69 & 576.65 & 1315.54 & 1566.38 \\
\hline & 1000 & 463.14 & 668.27 & 610.68 & 620.14 & 1405.14 & 1207.77 \\
\hline
\end{tabular}




$$
\text { "output" — 2019/2/14 — 3:53 — page } 16 \text { — \#16 }
$$
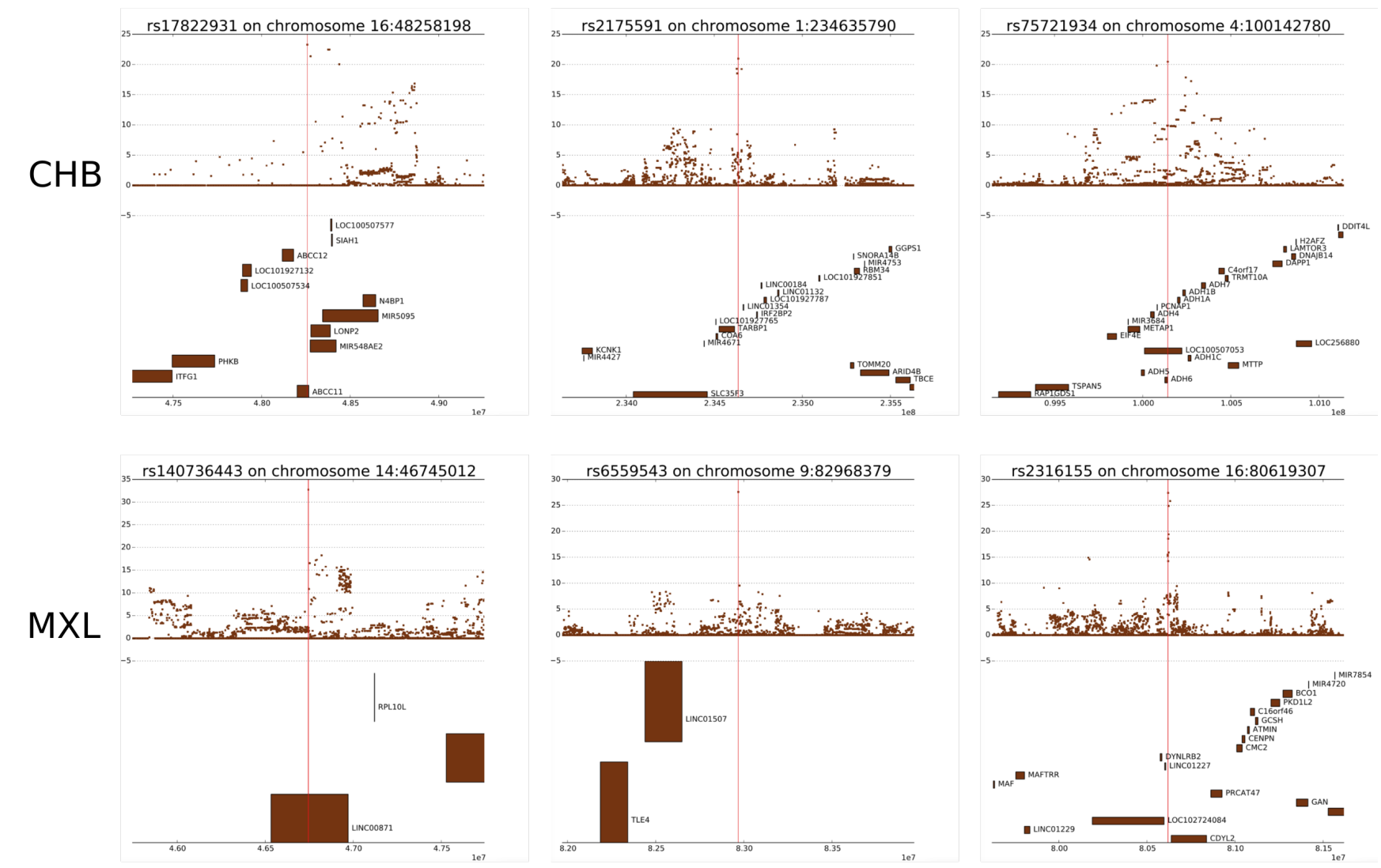

YRI
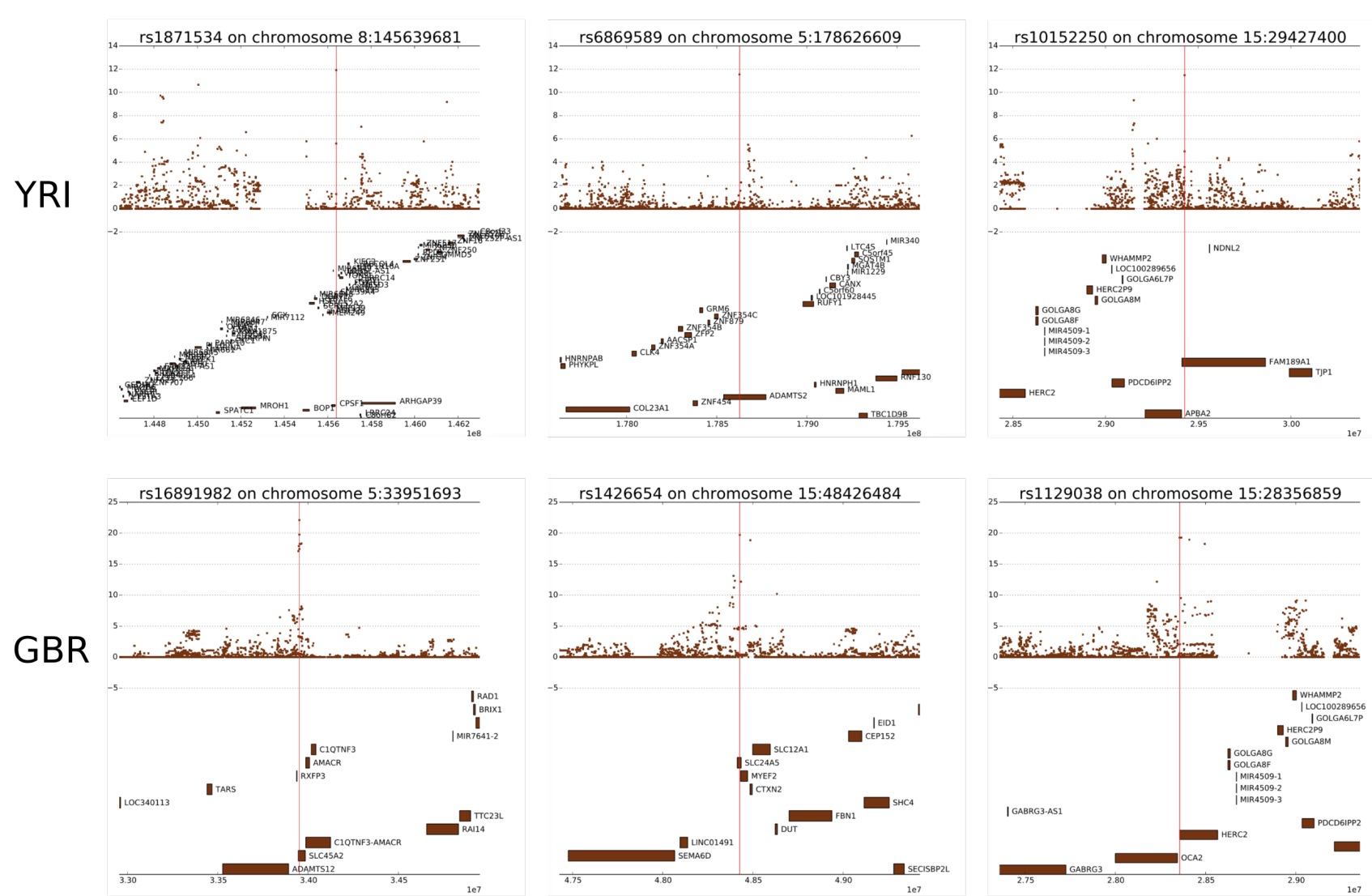

FIG. 6. Top 5 annotated peaks in each of the ancestry-specific selection studies. MXL-specific $=$ scan for selection in Native American ancestry of MXL. GBR-specific = scan for selection in European ancestry of GBR. CHB-specific = scan for selection in East Asian ancestry of CHB. YRI-specific = scan for selection in Yoruba African ancestry or ancestral for selection in East Asian ancestry of CHB. YRI-specific = scan for selection in Yoruba African ancestry or ancestral
non-African ancestry. We analyzed 5,601,710 variable sites across the autosomal genomes. We inferred genome-wide allele frequencies and covariances as described in the Methods section. We applied a likelihood model for each SNP by rescaling all variances and covariances by a scalar multiplier $\alpha$. Descriptions of each candidate region are in Table 3 . 


$$
\text { "output" — 2019/2/14 — 3:53 — page } 17 \text { — \#17 }
$$

Ohana · doi:10.1093/molbev/mst012

MBE

Table 3. Top 10 most differentiated SNPs from each of the ancestry-specific scans. LLRS $=$ log-likelihood ratio score for positive selection.

\begin{tabular}{|c|c|c|c|c|c|}
\hline $\mathrm{chr}$ & pos & rsid & LLRS & target ancestry & nearest gene \\
\hline 5 & 33951693 & rs16891982 & 22.085902 & European & SLC45A2 \\
\hline 15 & 48426484 & rs1426654 & 19.707464 & European & SLC24A5 \\
\hline 15 & 28356859 & rs1129038 & 19.290553 & European & HERC2 \\
\hline 15 & 28495956 & rs12912427 & 18.270213 & European & HERC2 \\
\hline 9 & 16792200 & rs10962596 & 15.819739 & European & BNC2 \\
\hline 1 & 1385211 & rs1312568 & 15.066101 & European & ATAD3C \\
\hline 2 & 136407479 & rs1446585 & 14.957582 & European & R3HDM1 \\
\hline 2 & 136616754 & rs182549 & 14.629386 & European & MCM6 \\
\hline 1 & 204784969 & rs3940119 & 14.393216 & European & NFASC \\
\hline 4 & 38798648 & rs5743618 & 14.38681 & European & TLR1 \\
\hline $\begin{array}{l}16 \\
16\end{array}$ & $\begin{array}{l}48258198 \\
48375777\end{array}$ & $\begin{array}{l}\text { rs17822931 } \\
\text { rs6500380 }\end{array}$ & $\begin{array}{l}23.271759 \\
22.474103\end{array}$ & $\begin{array}{l}\text { East Asian } \\
\text { East Asian }\end{array}$ & $\begin{array}{l}\text { ABCC11 } \\
\text { LONP2 }\end{array}$ \\
\hline 1 & 234635790 & rs2175591 & 20.95541 & East Asian & TARBP1 \\
\hline 4 & 100142780 & rs75721934 & 20.453247 & East Asian & LOC100507053 \\
\hline 11 & 61579427 & rs72643557 & 20.114033 & East Asian & FADS1 \\
\hline 11 & 120154631 & rs12224052 & 19.696284 & East Asian & POU2F3 \\
\hline 21 & 43974948 & rs228088 & 19.518001 & East Asian & SLC37A1 \\
\hline 11 & 133043841 & rs79802711 & 19.157192 & East Asian & OPCML \\
\hline 5 & 128016573 & rs79478220 & 18.476104 & East Asian & FBN2 \\
\hline 19 & 51441759 & rs11084040 & 18.158963 & East Asian & KLK5 \\
\hline $\begin{array}{l}14 \\
9\end{array}$ & $\begin{array}{l}46745012 \\
82968379\end{array}$ & $\begin{array}{l}\text { rs } 140736443 \\
\text { rs6559543 }\end{array}$ & $\begin{array}{l}32.730697 \\
27.584847\end{array}$ & $\begin{array}{l}\text { Native American } \\
\text { Native American }\end{array}$ & $\begin{array}{l}\text { LINC00871 } \\
\text { LINC01507 }\end{array}$ \\
\hline 16 & 80619307 & rs2316155 & 27.399123 & Native American & LINC01227 \\
\hline 14 & 21647765 & rs77549780 & 27.355769 & Native American & LINC00641 \\
\hline 12 & 14189549 & rs12425115 & 25.867367 & Native American & GRIN2B \\
\hline 10 & 8150713 & rs10508343 & 25.609772 & Native American & GATA3 \\
\hline 15 & 34936250 & rs16959274 & 25.424824 & Native American & GOLGA8B \\
\hline 8 & 4490837 & rs71523639 & 24.59957 & Native American & CSMD1 \\
\hline 1 & 14301862 & rs72640512 & 24.455822 & Native American & PRDM2 \\
\hline 12 & 29817716 & rs12580697 & 23.967094 & Native American & TMTC1 \\
\hline 14 & 57318110 & rs75607199 & 23.959875 & Native American & OTX2-AS1 \\
\hline 8 & 145639681 & rs1871534 & 11.906794 & Yoruba / Ancestral Non-African & SLC39A4 \\
\hline 5 & 178626609 & rs6869589 & 11.541667 & Yoruba / Ancestral Non-African & ADAMTS2 \\
\hline 15 & 29427400 & rs10152250 & 11.48232 & Yoruba / Ancestral Non-African & FAM189A1 \\
\hline 1 & 1106112 & rs6670693 & 11.447873 & Yoruba / Ancestral Non-African & TTLL10 \\
\hline 4 & 3666494 & rs58827274 & 11.341367 & Yoruba / Ancestral Non-African & LOC100133461 \\
\hline 17 & 2631985 & rs4790359 & 11.118134 & Yoruba / Ancestral Non-African & PAFAH1B1 \\
\hline 9 & 136769888 & rs2789823 & 11.031687 & Yoruba / Ancestral Non-African & VAV2 \\
\hline 6 & 169656029 & rs6930377 & 10.824098 & Yoruba / Ancestral Non-African & THBS2 \\
\hline 17 & 29350769 & rs8073072 & 10.794224 & Yoruba / Ancestral Non-African & RNF135 \\
\hline 5 & 173642871 & rs10067518 & 10.787147 & Yoruba / Ancestral Non-African & HMP19 \\
\hline
\end{tabular}




$$
\text { "output" — 2019/2/14 — 3:53 — page } 18 \text { — \#18 }
$$

Cheng et al. · doi:10.1093/molbev/mst012

$\mathrm{MBE}$

\section{Acknowledgments}

The authors gratefully acknowledge Thomas Mailund, Mikkel Schierup, Christian Storm Pedersen, and the GenomeDK staff for their support during the course of this research. FR thanks the Villum Foundation for their support.

\section{References}

Voight, B.F., Kudaravalli, S., Wen, X. and Pritchard, J.K., 2006. A map of recent positive selection in the human genome. PLoS biology, $4(3)$, p.e72.

Sabeti, P.C., Varilly, P., Fry, B., Lohmueller, J., Hostetter, E., Cotsapas, C., Xie, X., Byrne, E.H., McCarroll, S.A., Gaudet, R. and Schaffner, S.F., 2007. Genome-wide detection and characterization of positive selection in human populations. Nature, 449(7164), p.913.

Wright, Sewall. The genetical structure of populations. Annals of eugenics 15(1), 323-354.

Weir, Bruce S., et al. Measures of human population structure show heterogeneity among genomic regions. Genome research 15(11), 14681476.

Kim, Y. and Stephan, W., 2002. Detecting a local signature of genetic hitchhiking along a recombining chromosome. Genetics, 160(2), pp.765-777.

Kim, Y. and Nielsen, R., 2004. Linkage disequilibrium as a signature of selective sweeps. Genetics, 167(3), pp.1513-1524.
McVean, G., 2007. The structure of linkage disequilibrium around a selective sweep. Genetics, 175(3), pp.1395-1406.

Tajima, F., 1989. Statistical method for testing the neutral mutation hypothesis by DNA polymorphism. Genetics, 123(3), pp.585-595.

Fu, Y.X. and Li, W.H., 1993. Statistical tests of neutrality of mutations. Genetics, 133(3), pp.693-709.

Fay, J.C. and Wu, C.I., 2000. Hitchhiking under positive Darwinian selection. Genetics, 155(3), pp.1405-1413.

Nielsen, R., 2005. Molecular signatures of natural selection. Annu. Rev. Genet., 39, pp.197-218.

Beaumont, M.A. and Nichols, R.A., 1996. Evaluating loci for use in the genetic analysis of population structure. Proc. R. Soc. Lond. B, 263(1377), pp.1619-1626.

Akey, J.M., Zhang, G., Zhang, K., Jin, L. and Shriver, M.D., 2002. Interrogating a highdensity SNP map for signatures of natural selection. Genome research, 12(12), pp.18051814.

Beaumont, M.A. and Balding, D.J., 2004. Identifying adaptive genetic divergence among populations from genome scans. Molecular ecology, 13(4), pp.969-980.

Yi, X., Liang, Y., Huerta-Sanchez, E., Jin, X., Cuo, Z.X.P., Pool, J.E., Xu, X., Jiang, 


$$
\text { "output" — 2019/2/14 — 3:53 — page } 19 \text { — \#19 }
$$

H., Vinckenbosch, N., Korneliussen, T.S. and Zheng, H., 2010. Sequencing of 50 human exomes reveals adaptation to high altitude. Science, 329(5987), pp.75-78.

Bonhomme, M., Chevalet, C., Servin, B., Boitard, S., Abdallah, J.M., Blott, S. and San Cristobal, M., 2010. Detecting selection in population trees: the Lewontin and Krakauer test extended. Genetics.

Fumagalli, M., Moltke, I., Grarup, N., Racimo, F., Bjerregaard, P., Jrgensen, M.E., Korneliussen, T.S., Gerbault, P., Skotte, L., Linneberg, A. and Christensen, C., 2015. Greenlandic Inuit show genetic signatures of diet and climate adaptation. Science, 349(6254), pp.1343-1347.

Racimo, F., Marnetto, D. and HuertaSanchez, E., 2016. Signatures of archaic adaptive introgression in present-day human populations. Molecular biology and evolution, 34(2), pp.296-317.

Racimo, F., 2016. Testing for ancient selection using cross-population allele frequency differentiation. Genetics, 202(2), pp.733-750.

Cheng, X., Xu, C. and DeGiorgio, M., 2017. Fast and robust detection of ancestral selective sweeps. Molecular ecology, 26(24), pp.68716891.

Librado, P. and Orlando, L., 2018. Detecting signatures of positive selection along defined branches of a population tree using LSD.
Molecular biology and evolution, 35(6), pp.1520-1535.

Gnther, T. and Coop, G., 2013. Robust identification of local adaptation from allele frequencies. Genetics, pp.genetics-113.

Gautier, M., 2015. Genome-wide scan for adaptive divergence and association with populationspecific covariates. Genetics, pp.genetics-115.

Fariello, M.I., Boitard, S., Naya, H., San Cristobal, M. and Servin, B., 2013. Detecting signatures of selection through haplotype differentiation among hierarchically structured populations. Genetics, pp.genetics-112.

Huber, C.D., DeGiorgio, M., Hellmann, I. and Nielsen, R., 2016. Detecting recent selective sweeps while controlling for mutation rate and background selection. Molecular ecology, 25(1), pp.142-156.

Foll, M. and Gaggiotti, O.E., 2008. A genome scan method to identify selected loci appropriate for both dominant and codominant markers: a Bayesian perspective. Genetics.

Foll, M., Gaggiotti, O.E., Daub, J.T., Vatsiou, A. and Excoffier, L., 2014. Widespread signals of convergent adaptation to high altitude in Asia and America. The American Journal of Human Genetics, 95(4), pp.394-407.

Mathieson, I., Lazaridis, I., Rohland, N., Mallick, S., Patterson, N., Roodenberg, S.A., Harney, 


$$
\text { "output" — 2019/2/14 — 3:53 — page } 20-\# 20
$$

Cheng et al. · doi:10.1093/molbev/mst012

$\mathrm{MBE}$

E., Stewardson, K., Fernandes, D., Novak, M. and Sirak, K., 2015. Genome-wide patterns of selection in 230 ancient Eurasians. Nature, 528(7583), p.499.

Cheng, J.Y., Mailund, T. and Nielsen, R., 2017. Fast admixture analysis and population tree estimation for SNP and NGS data. Bioinformatics, 33(14), pp.2148-2155.

Skotte, L., Korneliussen, T.S. and Albrechtsen, A., 2013. Estimating individual admixture proportions from next generation sequencing data. Genetics, pp.genetics-113.

Felsenstein, J., 1985. Phylogenies and the comparative method. The American Naturalist, 125(1), pp.1-15.

Ewing, G. and Hermisson, J., 2010. MSMS: a coalescent simulation program including recombination, demographic structure and selection at a single locus. Bioinformatics, 26(16), pp.2064-2065.

Rentzsch, Philipp, et al., 2018. CADD: predicting the deleteriousness of variants throughout the human genome. Nucleic acids research 47(D1), D886-D894.

Adzhubei, I., Jordan, D.M. and Sunyaev, S.R., 2013. Predicting functional effect of human missense mutations using PolyPhen2. Current protocols in human genetics, 76(1), pp.7-20.

Sim, N.L., Kumar, P., Hu, J., Henikoff, S., Schneider, G. and Ng, P.C., 2012. SIFT web server: predicting effects of amino acid substitutions on proteins. Nucleic acids research, 40(W1), pp.W452-W457.

Siepel, A., Bejerano, G., Pedersen, J.S., Hinrichs, A.S., Hou, M., Rosenbloom, K., Clawson, H., Spieth, J., Hillier, L.W., Richards, S. and Weinstock, G.M., 2005. Evolutionarily conserved elements in vertebrate, insect, worm, and yeast genomes. Genome research, 15(8), pp.1034-1050.

Sato, H., Miyamoto, T., Yogev, L., Namiki, M., Koh, E., Hayashi, H., Sasaki, Y., Ishikawa, M., Lamb, D.J., Matsumoto, N. and Birk, O.S., 2006. Polymorphic alleles of the human MEI1 gene are associated with human azoospermia by meiotic arrest. Journal of human genetics, 51(6), pp.533-540.

Lappalainen, T., Sammeth, M., Friedlnder, M.R., ACt Hoen, P., Monlong, J., Rivas, M.A., Gonzalez-Porta, M., Kurbatova, N., Griebel, T., Ferreira, P.G. and Barann, M., 2013. Transcriptome and genome sequencing uncovers functional variation in humans. Nature, 501(7468), p.506.

Lonsdale, J., Thomas, J., Salvatore, M., Phillips, R., Lo, E., Shad, S., Hasz, R., Walters, G., Garcia, F., Young, N. and Foster, B., 2013. The genotype-tissue expression (GTEx) project. Nature genetics, 45(6), p.580. 


$$
\text { "output" — 2019/2/14 — 3:53 — page } 21-\# 21
$$

Ohana · doi:10.1093/molbev/mst012

MBE

MacArthur, J., Bowler, E., Cerezo, M., Gil, L., Hall, P., Hastings, E., Junkins, H., McMahon, A., Milano, A., Morales, J. and Pendlington, Z.M., 2017. The new NHGRI-EBI Catalog of published genome-wide association studies (GWAS Catalog). Nucleic acids research, 45(D1), pp.D896-D901.

Pritchard, J.K., Stephens, M. and Donnelly, P., 2000. Inference of population structure using multilocus genotype data. Genetics, 155(2), pp.945-959.

Alexander, D.H., Novembre, J. and Lange, K., 2009. Fast model-based estimation of ancestry in unrelated individuals. Genome research.

Pickrell, J.K. and Pritchard, J.K., 2012. Inference of population splits and mixtures from genomewide allele frequency data. PLoS genetics, 8(11), p.e1002967.

Murty, K.G. and Yu, F.T., 1988. Linear complementarity, linear and nonlinear programming (Vol. 3, pp. 447-448). Berlin: Heldermann.

Nelder, J.A. and Mead, R., 1965. A simplex method for function minimization. The computer journal, 7(4), pp.308-313.

Kiefer, J., 1953. Sequential minimax search for a maximum. Proceedings of the American mathematical society, 4(3), pp.502-506.

Coop, G., Witonsky, D., Di Rienzo, A. and Pritchard, J.K., 2010. Using environmental correlations to identify loci underlying local adaptation. Genetics.

Cholesky, A.L., 1910. Sur la rsolution numrique des systmes dquations linaires. Bulletin de la Sabix. Socit des amis de la Bibliothque et de l'Histoire de l'cole polytechnique, (39), pp.8195.

Self, S.G. and Liang, K.Y., 1987. Asymptotic properties of maximum likelihood estimators and likelihood ratio tests under nonstandard conditions. Journal of the American Statistical Association, 82(398), pp.605-610.

1000 Genomes Project Consortium, 2015. A global reference for human genetic variation. Nature, 526(7571), p.68.

DeGiorgio, M., Huber, C.D., Hubisz, M.J., Hellmann, I. and Nielsen, R., 2016. SweepFinder2: increased sensitivity, robustness and flexibility. Bioinformatics, 32(12), pp.1895-1897.

Sabeti, P.C., Schaffner, S.F., Fry, B., Lohmueller, J., Varilly, P., Shamovsky, O., Palma, A., Mikkelsen, T.S., Altshuler, D. and Lander, E.S., 2006. Positive natural selection in the human lineage. science, 312(5780), pp.1614-1620.

Duforet-Frebourg, N., Luu, K., Laval, G., Bazin, E. and Blum, M.G., 2015. Detecting genomic signatures of natural selection with principal component analysis: application to 
the 1000 Genomes data. Molecular biology and evolution, 33(4), pp.1082-1093.

Branicki, W., Brudnik, U., Draus-Barini, J., Kupiec, T. and Wojas-Pelc, A., 2008. Association of the SLC45A2 gene with physiological human hair colour variation. Journal of human genetics, 53(11-12), p.966.

Kimura, R., Yamaguchi, T., Takeda, M., Kondo, O., Toma, T., Haneji, K., Hanihara, T., Matsukusa, H., Kawamura, S., Maki, K. and Osawa, M., 2009. A common variation in EDAR is a genetic determinant of shovel-shaped incisors. The American Journal of Human Genetics, 85(4), pp.528-535.

Lamason, R.L., Mohideen, M.A.P., Mest, J.R., Wong, A.C., Norton, H.L., Aros, M.C., Jurynec, M.J., Mao, X., Humphreville, V.R., Humbert, J.E. and Sinha, S., 2005. SLC24A5, a putative cation exchanger, affects pigmentation in zebrafish and humans. Science, 310(5755), pp.1782-1786.

Sidiropoulos, N., Sohi, S.H., Pedersen, T.L., Porse, B.T., Winther, O., Rapin, N. and Bagger, F.O., 2018. SinaPlot: an enhanced chart for simple and truthful representation of single observations over multiple classes. Journal of Computational and Graphical Statistics, pp.14.

Hoffman, M.M., Buske, O.J., Wang, J., Weng, Z., Bilmes, J.A. and Noble, W.S.,
2012. Unsupervised pattern discovery in human chromatin structure through genomic segmentation. Nature methods, 9(5), p.473.

Canela-Xandri, O., Rawlik, K. and Tenesa, A., 2018. An atlas of genetic associations in UK Biobank. Nature Genetics, 50(11), p.1593.

Davydov, E.V., Goode, D.L., Sirota, M., Cooper, G.M., Sidow, A. and Batzoglou, S., 2010. Identifying a high fraction of the human genome to be under selective constraint using GERP++. PLoS computational biology, 6(12), p.e1001025.

Siepel, A., Bejerano, G., Pedersen, J.S., Hinrichs, A.S., Hou, M., Rosenbloom, K., Clawson, H., Spieth, J., Hillier, L.W., Richards, S. and Weinstock, G.M., 2005. Evolutionarily conserved elements in vertebrate, insect, worm, and yeast genomes. Genome research, 15(8), pp.1034-1050.

Pollard, K.S., Hubisz, M.J., Rosenbloom, K.R. and Siepel, A., 2010. Detection of nonneutral substitution rates on mammalian phylogenies. Genome research, 20(1), pp.110-121.

Mathieson, I., Lazaridis, I., Rohland, N., Mallick, S., Patterson, N., Roodenberg, S.A., Harney, E., Stewardson, K., Fernandes, D., Novak, M. and Sirak, K., 2015. Genome-wide patterns of selection in 230 ancient Eurasians. Nature, 528(7583), p.499. 
Bersaglieri, T., Sabeti, P.C., Patterson, N., Vanderploeg, T., Schaffner, S.F., Drake, J.A., Rhodes, M., Reich, D.E. and Hirschhorn, J.N., 2004. Genetic signatures of strong recent positive selection at the lactase gene. The American Journal of Human Genetics, 74(6), pp.1111-1120.

Voight, B.F., Kudaravalli, S., Wen, X. and Pritchard, J.K., 2006. A map of recent positive selection in the human genome. PLoS biology, $4(3)$, p.e72.

Vernot, B. and Akey, J.M., 2014. Resurrecting surviving Neandertal lineages from modern human genomes. Science, p.1245938.

Barreiro, L.B., Ben-Ali, M., Quach, H., Laval, G., Patin, E., Pickrell, J.K., Bouchier, C., Tichit, M., Neyrolles, O., Gicquel, B. and Kidd, J.R., 2009. Evolutionary dynamics of human Toll-like receptors and their different contributions to host defense. PLoS genetics, 5(7), p.e1000562.

Liu, S., Huang, S., Chen, F., Zhao, L., Yuan, Y., Francis, S.S., Fang, L., Li, Z., Lin, L., Liu, R. and Zhang, Y., 2018. Genomic analyses from non-invasive prenatal testing reveal genetic associations, patterns of viral infections, and Chinese population history. Cell, 175(2), pp.347-359.

Refoyo-Martnez, A., da Fonseca, R.R., Halldrsdttir, K., rnason, E., Mailund, T. and Racimo, F., 2018. Identifying loci under positive selection in complex population histories. bioRxiv, p.453092.

Ohashi, J., Naka, I., Tsuchiya, N., 2011. The impact of natural selection on an ABCC11 SNP determining earwax type. Mol. Biol. Evol. 28, 849-857

Peng, Y., Shi, H., Qi, X.B., Xiao, C.J., Zhong, H., Run-lin, Z.M. and Su, B., 2010. The ADH1B Arg47His polymorphism in East Asian populations and expansion of rice domestication in history. BMC evolutionary biology, 10(1), p.15.

Refoyo-Martínez, Alba, et al., 2018. Identifying loci under positive selection in complex population histories. bioRxiv, 453092.

Endo, Chihiro, et al., 2018. Genome-wide association study in Japanese females identifies fifteen novel skin-related trait associations. Scientific reports 8 .

Engelken, J., Carnero-Montoro, E., Pybus, M., Andrews, G.K., Lalueza-Fox, C., Comas, D., Sekler, I., de la Rasilla, M., Rosas, A., Stoneking, M. and Valverde, M.A., 2014. Extreme population differences in the human zinc transporter ZIP4 (SLC39A4) are explained by positive selection in Sub-Saharan Africa. PLoS genetics, 10(2), p.e1004128.

Barban, N., Jansen, R., De Vlaming, R., Vaez, A., Mandemakers, J.J., Tropf, F.C., Shen, X., Wilson, J.F., Chasman, D.I., Nolte, 
I.M. and Tragante, V., 2016. Genome-wide analysis identifies 12 loci influencing human reproductive behavior. Nature genetics, 48(12), p.1462.

Zeng, Y., Nie, C., Min, J., Chen, H., Liu, X., Ye, R., Chen, Z., Bai, C., Xie, E., Yin, Z. and Lv, Y., 2018. Sex differences in genetic associations with longevity. JAMA network open, 1(4), pp.e181670-e181670.

Kaufman, C.K., Zhou, P., Pasolli, H.A., Rendl, M., Bolotin, D., Lim, K.C., Dai, X., Alegre, M.L. and Fuchs, E., 2003. GATA3: an unexpected regulator of cell lineage determination in skin. Genes development, 17(17), pp.2108-2122.

Yang, J.J., Cheng, C., Yang, W., Pei, D., Cao, X., Fan, Y., Pounds, S.B., Neale, G., Trevio, L.R., French, D. and Campana, D., 2009. Genomewide interrogation of germline genetic variation associated with treatment response in childhood acute lymphoblastic leukemia. Jama, 301(4), pp.393-403.

Eystathioy, T., Jakymiw, A., Fujita, D.J., Fritzler, M.J. and Chan, E.K., 2000. Human autoantibodies to a novel Golgi protein golgin67: high similarity with golgin-95/gm 130 autoantigen. Journal of autoimmunity, 14(2), pp.179-187.

Sunryd, J.C., Cheon, B., Graham, J.B., Giorda, K.M., Fissore, R.A. and Hebert, D.N., 2014.
TMTC1 and TMTC2 are novel endoplasmic reticulum TPR-containing adapter proteins involved in calcium homeostasis. Journal of Biological Chemistry, pp.jbc-M114.

Ragge, N.K., Brown, A.G., Poloschek, C.M., Lorenz, B., Henderson, R.A., Clarke, M.P., Russell-Eggitt, I., Fielder, A., Gerrelli, D., Martinez-Barbera, J.P. and Ruddle, P., 2005. Heterozygous mutations of OTX2 cause severe ocular malformations. The American Journal of Human Genetics, 76(6), pp.1008-1022.

Vincent, A., Forster, N., Maynes, J.T., Paton, T.A., Billingsley, G., Roslin, N.M., Ali, A., Sutherland, J., Wright, T., Westall, C.A. and Paterson, A.D., 2014. OTX2 mutations cause autosomal dominant pattern dystrophy of the retinal pigment epithelium. Journal of medical genetics, 51(12), pp.797-805.

Diaczok, D., Romero, C., Zunich, J., Marshall, I. and Radovick, S., 2008. A novel dominant negative mutation of OTX2 associated with combined pituitary hormone deficiency. The Journal of Clinical Endocrinology Metabolism, 93(11), pp.4351-4359.

Boncinelli, E., Gulisano, M. and Broccoli, V., 1993. Emx and Otx homeobox genes in the developing mouse brain. Journal of neurobiology, 24(10), pp.1356-1366.

Nishida, A., Furukawa, A., Koike, C., Tano, Y., Aizawa, S., Matsuo, I. and Furukawa, 
T., 2003. Otx2 homeobox gene controls retinal photoreceptor cell fate and pineal gland development. Nature neuroscience, 6(12), p. 1255 .

Peña, C.J., Kronman, H.G., Walker, D.M., Cates, H.M., Bagot, R.C., Purushothaman, I., Issler, O., Loh, Y.H.E., Leong, T., Kiraly, D.D. and Goodman, E., 2017. Early life stress confers lifelong stress susceptibility in mice via ventral tegmental area OTX2. Science, 356(6343), pp.1185-1188.

Sénéchal, A., Humbert, G., Surget, M.O., Bazalgette, C., Bazalgette, C., Arnaud, B., Arndt, C., Laurent, E., Brabet, P. and Hamel, C.P., 2006. Screening genes of the retinoid metabolism: novel LRAT mutation in leber congenital amaurosis. American journal of ophthalmology, 142(4), pp.702-704.

Thompson, D.A., Li, Y., McHenry, C.L., Carlson, T.J., Ding, X., Sieving, P.A., Apfelstedt-Sylla, E. and Gal, A., 2001. Mutations in the gene encoding lecithin retinol acyltransferase are associated with early-onset severe retinal dystrophy. Nature genetics, 28(2), p.123.

Otto, E.A., Loeys, B., Khanna, H., Hellemans, J., Sudbrak, R., Fan, S., Muerb, U., O’Toole, J.F., Helou, J., Attanasio, M. and Utsch, B., 2005. Nephrocystin-5, a ciliary IQ domain protein, is mutated in Senior-Loken syndrome and interacts with RPGR and calmodulin. Nature genetics, 37(3), p.282.
Kim, H., Song, K.D., Kim, H.J., Park, W., Kim, J., Lee, T., Shin, D.H., Kwak, W., Kwon, Y.J., Sung, S. and Moon, S., 2015. Exploring the genetic signature of body size in Yucatan miniature pig. PloS one, 10(4), p.e0121732.

Kwon, D.J., Lee, Y.S., Shin, D., Won, K.H. and Song, K.D., 2018. Genome analysis of Yucatan miniature pigs to assess their potential as biomedical model animals. Asian-Australasian journal of animal sciences, 32(2), pp.290-296.

Adly, N., Alhashem, A., Ammari, A. and Alkuraya, F.S., 2014. Ciliary Genes TBC 1 D 32/C6orf170 and SCLT 1 are Mutated in Patients with OFD Type IX. Human mutation, 35(1), pp.36-40.

Ko, H.W., Norman, R.X., Tran, J., Fuller, K.P., Fukuda, M. and Eggenschwiler, J.T., 2010. Broad-minded links cell cycle-related kinase to cilia assembly and hedgehog signal transduction. Developmental cell, 18(2), pp.237-247.

Jin, Y., Birlea, S.A., Fain, P.R., Ferrara, T.M., Ben, S., Riccardi, S.L., Cole, J.B., Gowan, K., Holland, P.J., Bennett, D.C. and Luiten, R.M., 2012. Genome-wide association analyses identify 13 new susceptibility loci for generalized vitiligo. Nature genetics, 44(6), p.676.

Nagel, M., Jansen, P.R., Stringer, S., Watanabe, K., de Leeuw, C.A., Bryois, J., Savage, J.E., 


$$
\text { "output" — 2019/2/14 — 3:53 — page } 26 \text { — \#26 }
$$

Cheng et al. · doi:10.1093/molbev $/ \mathrm{mst} 012$

Hammerschlag, A.R., Skene, N.G., Muoz-

Manchado, A.B. and White, T., 2018. Metaanalysis of genome-wide association studies for neuroticism in 449,484 individuals identifies novel genetic loci and pathways. Nature Genetics, 50(7), p.920.

Yoshiura, Koh-ichiro, et al. A SNP in the ABCC11 gene is the determinant of human earwax type. Nature genetics 38(3), 324 . 